\title{
Utilizing Fullerenols as Surfactant for Carbon Nanotubes Dispersions Preparation
}

\author{
Jianlong Ji, ${ }^{1,2}$ Chang Qiao, ${ }^{1}$ Yali Liu, ${ }^{1,2}$ Wendong Zhang, ${ }^{1}$ Shengbo Sang, \\ Xing Yang, ${ }^{3}$ Aoqun Jian, ${ }^{1}$ Qianqian Duan, ${ }^{1}$ Qiang Zhang, ${ }^{1}$ and Ying Liu ${ }^{4}$ \\ ${ }^{1}$ Micro Nano System Research Center, Key Lab of Advanced Transducers and Intelligent Control System of the Ministry of Education, \\ College of Information Engineering, Taiyuan University of Technology, Taiyuan 030024, China \\ ${ }^{2}$ Advanced Coal Mine Machinery and Equipment Collaborative Innovation Center of Shanxi Province, \\ College of Information Engineering, Taiyuan University of Technology, Taiyuan 030024, China \\ ${ }^{3}$ MEMS Laboratory, Department of Precision Instruments and Mechanology, Tsinghua University, Beijing 100084, China \\ ${ }^{4}$ College of Materials Science and Engineering, Taiyuan University of Technology, Taiyuan 030024, China
}

Correspondence should be addressed to Shengbo Sang; sangshengbo@tyut.edu.cn and Ying Liu; liuyingtyut@163.com

Received 30 September 2016; Accepted 10 January 2017; Published 23 February 2017

Academic Editor: Te-Hua Fang

Copyright (C) 2017 Jianlong Ji et al. This is an open access article distributed under the Creative Commons Attribution License, which permits unrestricted use, distribution, and reproduction in any medium, provided the original work is properly cited.

Dispersions of individual carbon nanotubes (CNTs) are crucial for nanodevices and polymer/CNTs nanocomposites. In this paper, stable and homogenous dispersions of individual multiwalled carbon nanotubes (MWCNTs) have been synthesized. The factors influencing the dispersibility mechanism, including the surfactant concentration and the $\mathrm{pH}$ value, have been investigated. SEM images display the impurities sticking on MWCNTs which have been removed. The oxygen-containing groups on the surface of MWCNTs sample have been detected through FT-IR and Raman spectra. All experimental results illustrate that using fullerenols as surfactant can greatly improve the dispersibility of MWCNTs. Moreover, the prepared dispersions exhibit good stability that the sediment percentage of fullerenols-MWCNTs is only $5.2 \%$ after 5 days.

\section{Introduction}

As a new type of one-dimensional nanomaterials, CNTs have attracted considerable attention due to its novel structure and unique physical and chemical properties in recent years [13]. The method to improve the dispersibility of individual CNTs is urgently requested because of the demand for high performance devices, such as transistors, sensors, and oscillators [4-8].

Generally, CNTs can be synthesized by arc discharge, laser ablation, and chemical vapor deposition (CVD), which inevitably contained amorphous carbon, carbon nanoparticles (CNPs), and metal catalyst particles [1,9-11]. To date, various methods to rarefy MWCNTs, including physical and chemical purification, have been explored. However, physical methods are always complicated and less effective, whereas the chemical methods usually destroy the MWCNTs' structure, especially one-step liquid phase oxidation $[12,13]$. Therefore, two-step liquid phase oxidation, which causes less damage to MWCNTs, has been exploited extensively over the recent years.

In most common solvents, van der Waals' force holds MWCNTs together and severely impedes its manipulation and application. To resolve this problem, various types of surfactants have been proposed to improve the dispersion properties, such as sodium dodecylbenzene sulfonate $(\mathrm{NaD}-$ DBs) whose dispersibility is limited and polyvinylpyrrolidone (PVP) which is hard to be removed [14, 15]. Besides, fullerenols exhibit unique structure where polar hydroxyl groups bond on the carbon cages and possess strong electronic adsorption capacity. This characteristic induces the physically adsorption of fullerenols onto the sidewall of MWCNTs by $\pi-\pi$ interactions, which enhances the dispersions homogeneity and stability significantly [16]. 


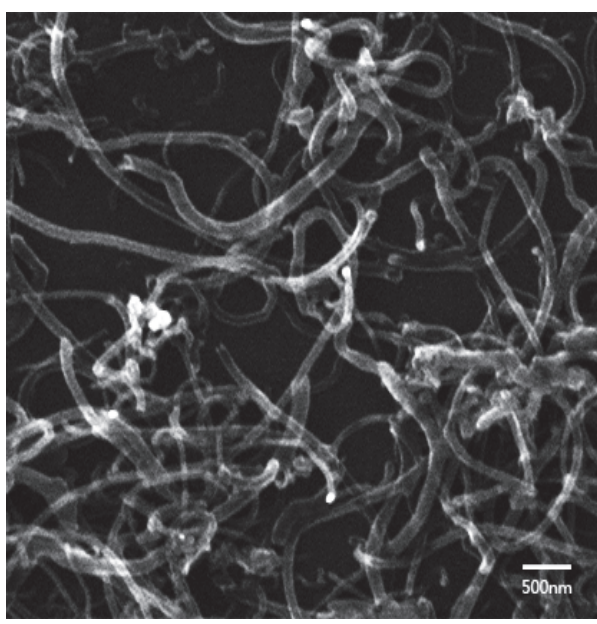

(a)

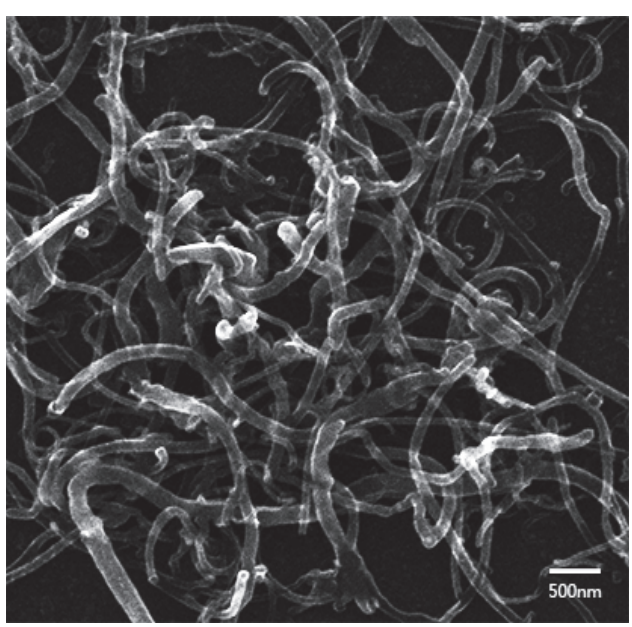

(b)

Figure 1: SEM images of MWCNTs: (a) R-MWCNTs; (b) P-MWCNTs.

In this work, MWCNTs were purified by two-step liquid phase oxidation with $\mathrm{NaOH}$ and a mixture of nitric and sulfuric acids $\left(\mathrm{H}_{2} \mathrm{SO}_{4} / \mathrm{HNO}_{3}\right.$, volume $\left.1: 3\right)$. Fullerenols serving as surfactants were used to disperse MWCNTs. The effects of fullerenols concentration and solution $\mathrm{pH}$ on the dispersibility of MWCNTs were investigated, respectively. Based on the experimental results, a possible mechanism of improvement of dispersibility was proposed.

\section{Materials and Methods}

2.1. Materials. The N-doped MWCNTs produced by chemical vapor deposition (CVD) were supplied by Chengdu Organic Chemicals Co. Ltd., Chinese Academy of Sciences. Fullerenes C60 were purchased from Suzhou Dade Carbon Nano Science and Technology Co. Ltd. NaDDBs and PVP were bought from Aladdin Reagent. All other reagents were of analytical reagent (AR) pure grade and were purchased from Sinopharm Chemical Reagent Co. Ltd.

2.2. Preparation of $M W C N T s$ Dispersions. To obtain the purified MWCNTs (P-MWCNTs), raw MWCNTs (R-MWCNTs) were refluxed in $2 \mathrm{~mol} / \mathrm{L} \mathrm{NaOH}$ for $12 \mathrm{~h}$. Then, MWCNTs were sonicated ( $400 \mathrm{~W}, \mathrm{KQ}-500 \mathrm{DE})$ for $30 \mathrm{~min}$ in a mixture of nitric/sulfuric acids (volume 1:3) and washed with deionized water until the eluate reached neutral. Finally, P-MWCNTs were dried at $80^{\circ} \mathrm{C}$ under vacuum.

The fullerenols were synthesized from powdered Fullerenes $\mathrm{C} 60$ by a novel method reported by Chiang et al. [17]. For preparing the MWCNTs dispersions, $25 \mathrm{mg}$ P-MWCNTs and $5 \mathrm{mg}$ surfactants (fullerenols, NaDDBs, and PVP) were sonicated for $30 \mathrm{~min}$ in $10 \mathrm{ml}$ methanol, respectively. Then, the stable dispersions were collected by centrifuging separation (3000 rpm, TG16-WS) to remove sediment and impurities underlying. Finally, dispersion 1, dispersion 2, and dispersion 3 of MWCNTs were obtained, respectively. Furthermore, the dispersion 1-1, dispersion 1-2, and dispersion 1-3 were prepared based on dispersion 1, in which the amount of fullerenols was adjusted by $2.5 \mathrm{mg}, 5 \mathrm{mg}$, and $10 \mathrm{mg}$, respectively.

2.3. Characterization. The morphologies and chemical compositions of the P-MWCNTs were characterized by scanning electron microscopy (SEM, JEOL JSM-7001F), atomic force microscope (AFM, Bruker Veeco Dimension-5), and Fourier transform infrared spectrometer (FT-IR, Bruker Tensor 27).

The optical absorbance of dispersions was measured by UV-Vis spectrum (METASH UV-8000A), and then the suspensions were collected, dried, and weighed to obtain the quality and concentration. Standard spectra of optical absorbance and the MWCNTs concentration can be obtained since the dispersion concentration is proportional to the dispersion absorbance.

\section{Results and Discussion}

3.1. Morphologies and Compositions Analysis. Figure 1 shows SEM images of R-MWCNTs and P-MWCNTs. R-MWCNTs with impurities, including amorphous carbon and metal particles, exist in large bundles (Figure 1(a)). P-MWCNTs contained fewer inclusions (Figure 1(b)) than R-MWCNTs since the metal particles can be dissolved by acid and amorphous carbon used to be oxidized under gentle conditions. The P-MWCNTs are prepared by sonication of R-MWCNTs in the binary mixture of $\mathrm{H}_{2} \mathrm{SO}_{4} / \mathrm{HNO}_{3}$ acid. Therefore, $\mathrm{P}$ MWCNTs possess more intrinsic properties considering that less damage has been brought to MWCNTs than refluxing in the mixed acid [18].

Figure 2 shows FT-IR spectra of R-MWCNTs and PMWCNTs. For the FT-IR spectrum of R-MWCNTs in Figure 2(a), the weak peaks at $3752 \mathrm{~cm}^{-1}$ and $690 \mathrm{~cm}^{-1}$ are attributed to the stretching vibration mode and rocking 


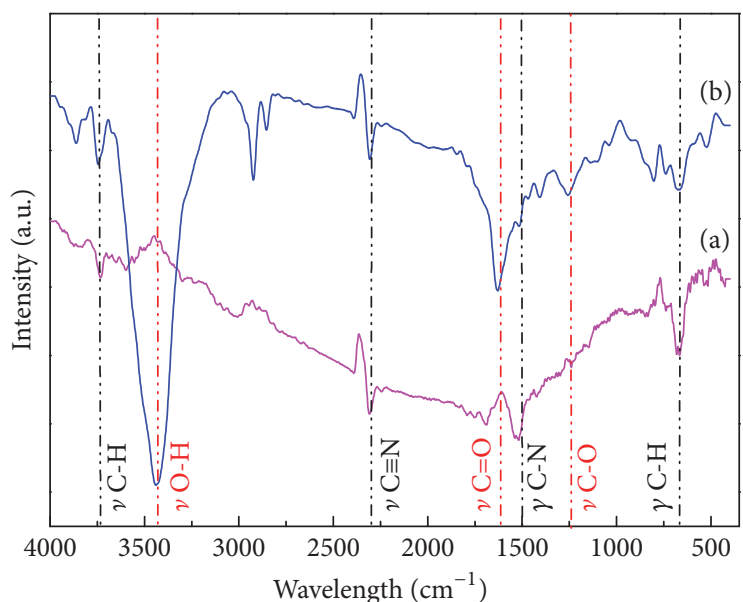

Figure 2: FT-IR spectra of (a) R-MWCNTs; (b) P-MWCNTs.

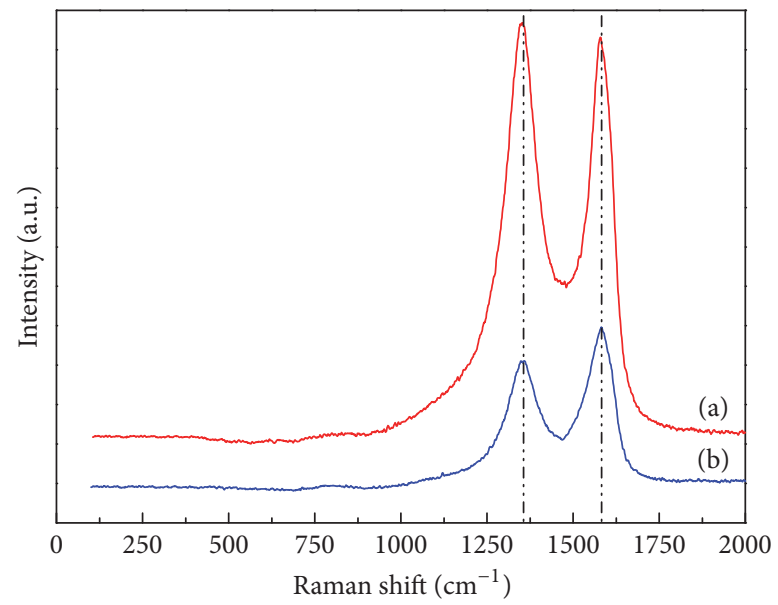

FIgURE 3: Raman spectra of (a) R-MWCNTs; (b) P-MWCNTs.

vibration mode of $\mathrm{C}-\mathrm{H}$, while the peaks which locate at $1500 \mathrm{~cm}^{-1}$ and $2300 \mathrm{~cm}^{-1}$ can be ascribed to the C-N rocking vibration and $\mathrm{C} \equiv \mathrm{N}$ stretching vibration in $\mathrm{N}$-doped $\mathrm{R}$ MWCNTs, respectively. Compared with the R-MWCNTs, several different absorption peaks are appeared in the FTIR spectrum of P-MWCNTs, as shown in Figure 2(b). Considering the influence of water has been already eliminated, the broad peak which appears at $3430 \mathrm{~cm}^{-1}$ is ascribed to the $\mathrm{O}-\mathrm{H}$ stretching vibration modes. The peak which appears at $1620 \mathrm{~cm}^{-1}$ is related to $\mathrm{C}=\mathrm{O}$ stretching in carboxyl groups, whereas the increased strength of the peak at $1250 \mathrm{~cm}^{-1}$ may be associated with C-O stretching in phenols [19]. The FTIR spectra indicate that a two-step purification using $\mathrm{NaOH}$ mixed acid as a solvent is capable of enhancing MWCNTs' chemical reaction activity, which is beneficial to functionalize hydroxyl and carboxyl groups and improve the performance of dispersions.

Differences of Raman spectra between R-MWCNTs and P-MWCNTs are illustrated in Figure 3. The G-band $\left(1590 \mathrm{~cm}^{-1}\right)$ represents the in-plane bond-stretching motion

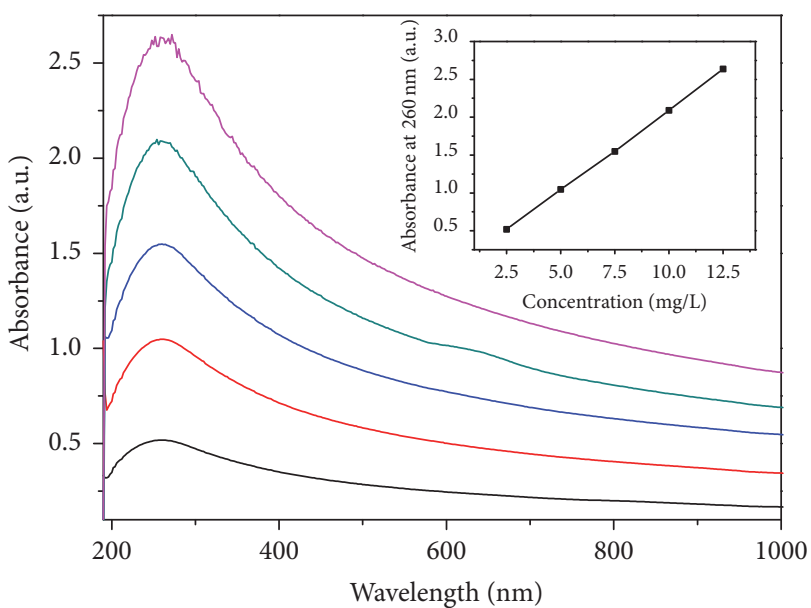

FIGURE 4: UV-Vis spectra of dispersion 1 with different concentrations in methanol; the inset illustrates the linear dependence of absorption intensity on suspension concentration.

of the pairs of $\mathrm{C} \mathrm{sp}{ }^{2}$ atoms ( $\mathrm{E}_{2 \mathrm{~g}}$ phonons), while the D-band $\left(1350 \mathrm{~cm}^{-1}\right)$ corresponds to breathing modes of rings or $\mathrm{K}$ point phonons of $A_{1 g}$ symmetry [20]. The structural integrity of the MWCNTs is characterized by ratio of D-band and Gband intensities $\left(I_{\mathrm{D}} / I_{\mathrm{G}}\right)$. The decrease of $I_{\mathrm{D}} / I_{\mathrm{G}}$ intensity ratio from 1.03 (R-MWCNTs) to 0.83 (P-MWCNTs) demonstrates that the purity of MWCNTs is improved obviously. Compared with R-MWCNTs, both the intensities of D-band and G-band of P-MWCNTs are reduced. The weakening of the Dband is due to the removal of carbonaceous impurities such as amorphous carbon and carbon nanoparticles [21]. The weakening of the G-band indicates that parts of defects are oxidized to hydrophilic groups such as hydroxyl and carboxyl [22], which are consistent with the results of FT-IR spectra.

3.2. Analysis of the Stability of Dispersions. Stability of the MWCNTs dispersion is of great importance in its applications. Dispersion 1 of P-MWCNTs was synthesized by using fullerenols as the surfactant. Figure 4 shows the UVVis absorbance spectra of the dispersion 1. Because of $1 \mathrm{D}$ van Hove singularities of individual CNTs [23], the characteristic bands of P-MWCNTs corresponding to an intense absorption peak at $260 \mathrm{~nm}$ can be clearly observed. Besides, the absorbance intensity is substantially linear in the concentration of dispersion 1 (the inset in Figure 4). The linear fashion indicates that a homogeneous suspension of fullerenols-MWCNTs is formed following the Beer-Lambert law.

The absorbance peak at $260 \mathrm{~nm}$ of all dispersions could characterize the concentration of MWCNTs seeing that the concentration of MWCNTs is proportional to the absorbance intensity of UV-Vis spectra. Generally, the sediment percentage used to characterize the suspension stability could be calculated by the following equation:

$$
S=\frac{A_{0}-A_{1}}{A_{0}},
$$




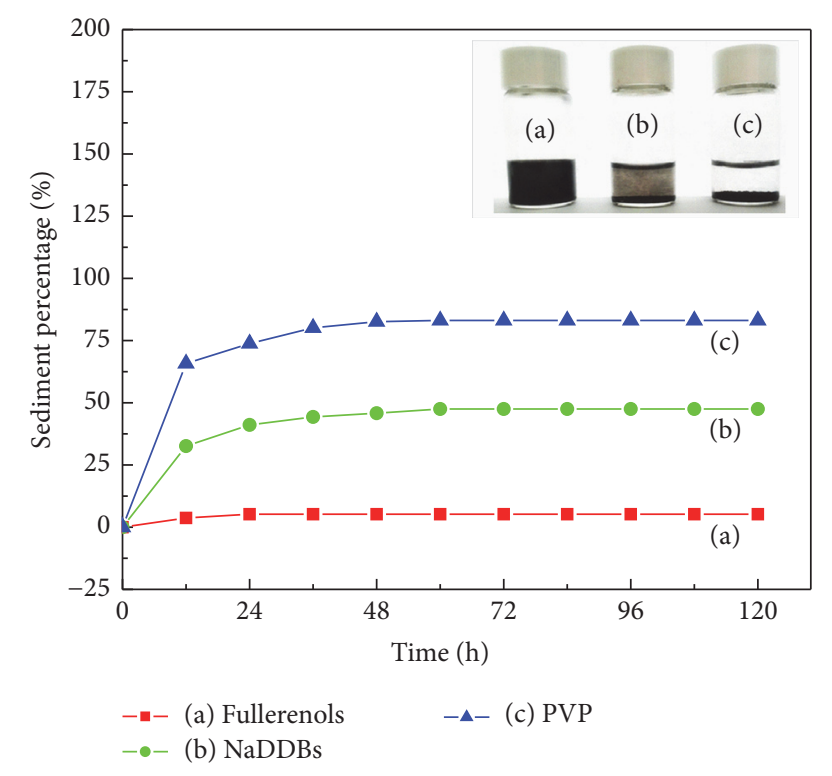

FIGURE 5: Variation of sediment percentage of dispersion 1, dispersion 2, and dispersion 3. The inserts are the optical images of P-MWCNTs dispersions with fullerenols, NaDDBs, and PVP after $120 \mathrm{~h}$ standing (from left to right), respectively.

where $A_{0}$ and $A_{1}$ are the absorbance intensity of the original solutions and the quiescent solutions. In our experiment, the sedimentation percentage was measured every 12 hours (Figure 5); the concentrations of surfactants including fullerenols, NaDDBs, and PVP were $500 \times 10^{-6} \mathrm{~g} / \mathrm{ml}$ for dispersion 1 , dispersion 2 , and dispersion 3 , whereas the concentrations of P-MWCNTs were about $1080 \times 10^{-6} \mathrm{~g} / \mathrm{ml}$, $720 \times 10^{-6} \mathrm{~g} / \mathrm{ml}$, and $530 \times 10^{-6} \mathrm{~g} / \mathrm{ml}$, respectively.

The results show that the sedimentation percentage of the dispersion 1 is the minimum, which is only $5.2 \%$ after 5 days standing. However, the sedimentation percentage of the dispersion 2 and dispersion 3 is $47.5 \%$ and $83.1 \%$, almost 10 and 20 times as much as that of dispersion 1. In right inset, it is also clearly observed that NaDDBS-MWCNTs and PVP-MWCNTs dispersions contain coagulated bundles at the bottom while fullerenols-MWCNTs dispersions are homogeneous.

\subsection{Dispersing Mechanism of P-MWCNTs with Fullerenols.} Fullerenols are soluble in polar solvents such as water and alcohol because of decorating of polar hydroxyl groups on the carbon cages. Further experimental results illustrated by SEM images in Figure 5 show that fullerenols can improve the dispersibility of P-MWCNTs significantly. Moreover, the cage structure of fullerenols would result in a strong capacity of electrons adsorption. Therefore, a physical adhesion between fullerenols and MWCNTs could be achieved by $\pi-\pi$ interaction, which can weaken the van der Waals force between MWCNTs and further improve the dispersibility and stability of MWCNTs [16].

In the experiment, the fullerenols concentrations in dispersion 1-1, dispersion 1-2, and dispersion 1-3 were 250 $\times 10^{-6} \mathrm{~g} / \mathrm{ml}, 500 \times 10^{-6} \mathrm{~g} / \mathrm{ml}$, and $1000 \times 10^{-6} \mathrm{~g} / \mathrm{ml}$, respectively. Accordingly, the concentrations of P-MWCNTs in the above dispersions were about $420 \times 10^{-6} \mathrm{~g} / \mathrm{ml}, 1080 \times$ $10^{-6} \mathrm{~g} / \mathrm{ml}$, and $1250 \times 10^{-6} \mathrm{~g} / \mathrm{ml}$. Figures $6(\mathrm{a})-6(\mathrm{c})$ show SEM images of dispersion 1-1, dispersion 1-2, and dispersion 1-3, respectively.

The quantity of P-MWCNTs which adheres to the substrate is proportional to the concentrations of fullerenols mentioned above. The phenomenon is consistent with the results of UV-Vis investigation. Figure 6(d) is the magnified SEM image of the area marked in Figure 6(b); it demonstrates that some individual P-MWCNTs have been taken from dispersion 1-2. More detailed information can be obtained from a quantitative AFM investigation (Figure 6(e)), which further shows the height profiles of an individual PMWCNT. The diameter is about $26.8 \mathrm{~nm}$ as illustrated in Figure 6(f).

The dispersibility also could be tuned by solutions $\mathrm{pH}$. In this work, the $\mathrm{pH}$ values of dispersion 1 have been adjusted by $\mathrm{HNO}_{3}$ and $\mathrm{NaOH}$. Figure 7 shows the SEM images of $\mathrm{P}$ MWCNTs of dispersions under acidic $(\mathrm{pH}=5)$ and alkaline $(\mathrm{pH}=9)$ conditions. It can be clearly observed that the dispersibility of P-MWCNTs in alkaline solution is better than that of the acid solution. These can be ascribed to two presumable reasons. Firstly, the dissociation of the oxygencontaining groups could lead to a rise in the concentration of negative charges on the surface of P-MWCNTs. This enhances the electrostatic repulsion between adjacent P-MWCNTs and results in a great improvement of dispersibility. Inversely, the dissociation is usually suppressed in the acidic condition. Secondly, the oxidations of fullerenes can readily proceed under alkaline condition and contribute to the oxygen-containing groups in the functionalization reaction. Nevertheless, this process is difficult to occur under acidic and neutral conditions because of the high energy barriers [24].

\section{Conclusions}

P-MWCNTs have been produced using two-step liquid phase oxidation treatment with $\mathrm{NaOH}$ and a mixture of nitric and sulfuric acids (1:3 by volume). SEM images have shown that oxidative treatment could remove the impurities in MWCNTs. Moreover, hydroxyl groups and carboxyl groups have also been identified on the surface of MWCNTs applying FT-IR and Raman spectroscopy. The results indicated that an effective route has been developed to disperse P-MWCNTs in methanol using the fullerenols as a surfactant. The conspicuously enhanced homogeneity and stability of dispersions can be attributed to excellent electronic absorption ability and weak acid dissociation properties of fullerenols in the solution. This approach of preparing dispersions of MWCNTs is promising to be used in the area of nanodevices and polymer/CNTs nanocomposites fabrication.

\section{Competing Interests}

The authors declare that they have no competing interests. 


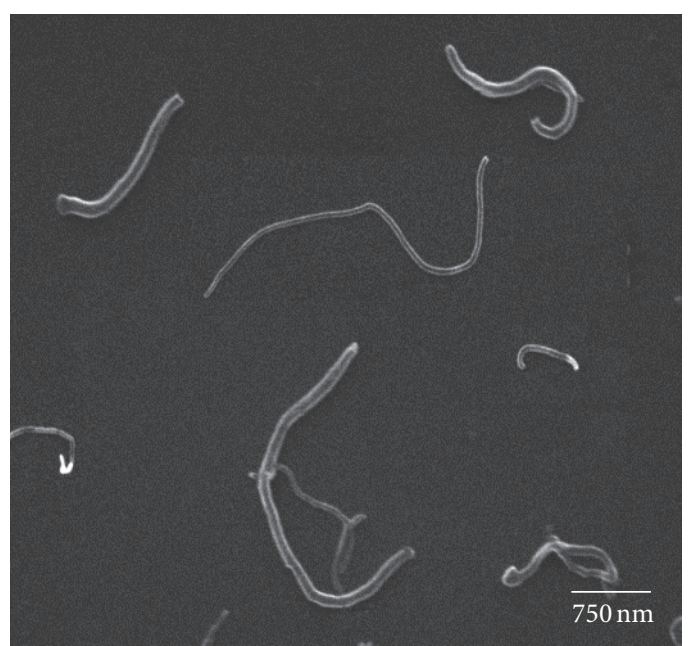

(a)

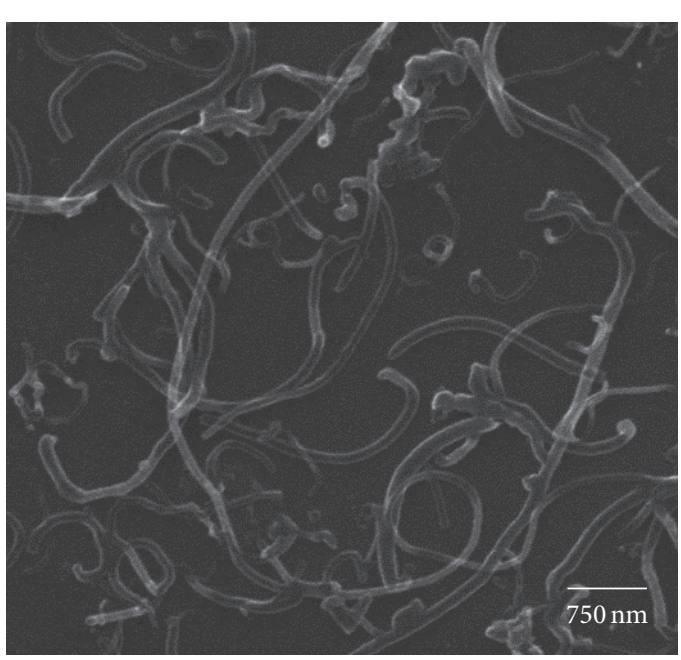

(c)

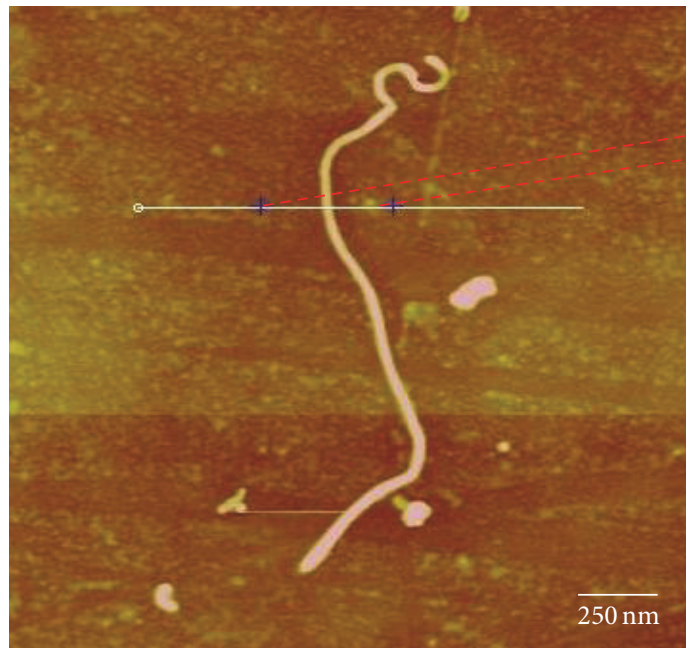

(e)

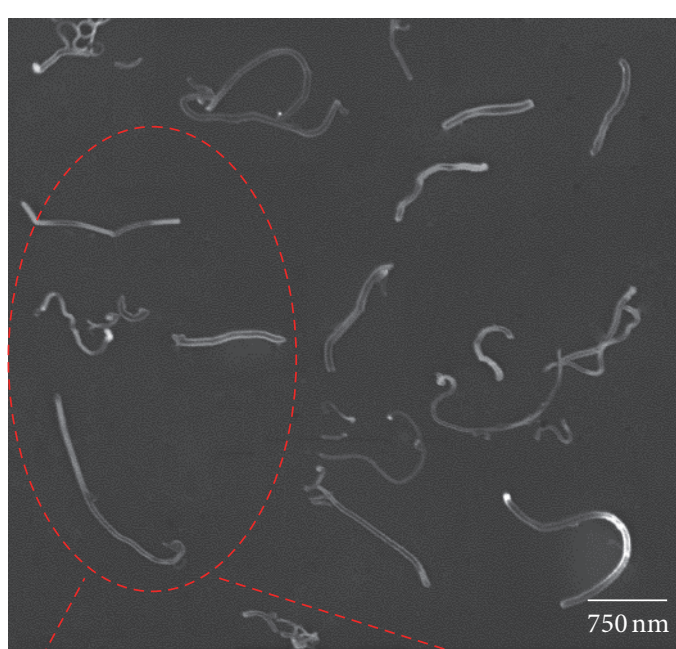

(b)

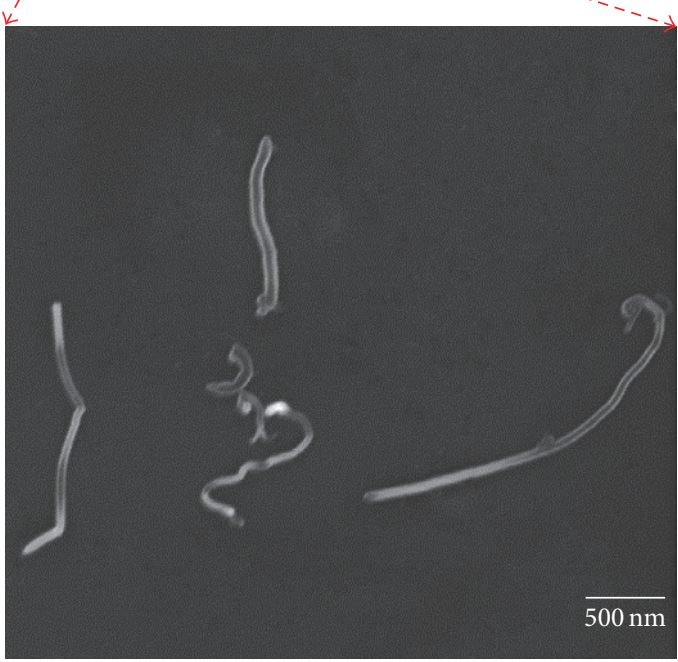

(d)

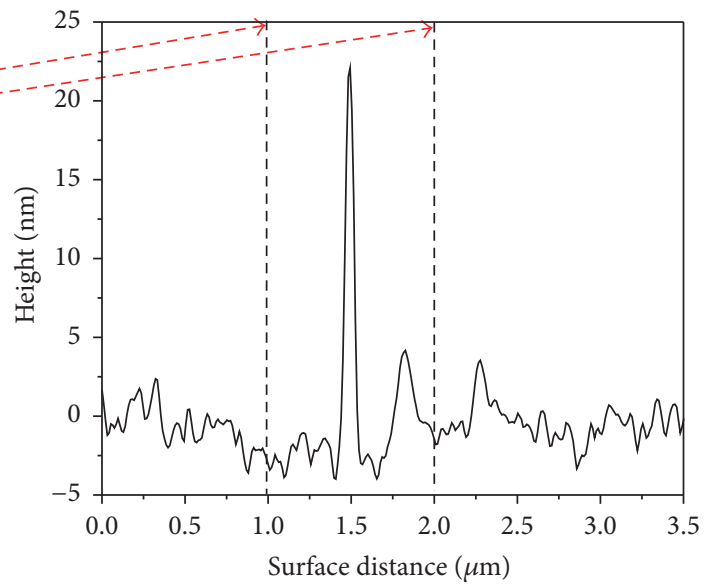

(f)

FIGURE 6: (a) SEM image of dispersion 1-1; (b) FESEM image of dispersion 1-2; (c) FESEM image of dispersion 1-3; (d) a further magnified SEM image from the area marked in image (b); (e) AFM image of dispersion 1-2; (f) cross sections along the solid lines in the image (e). 


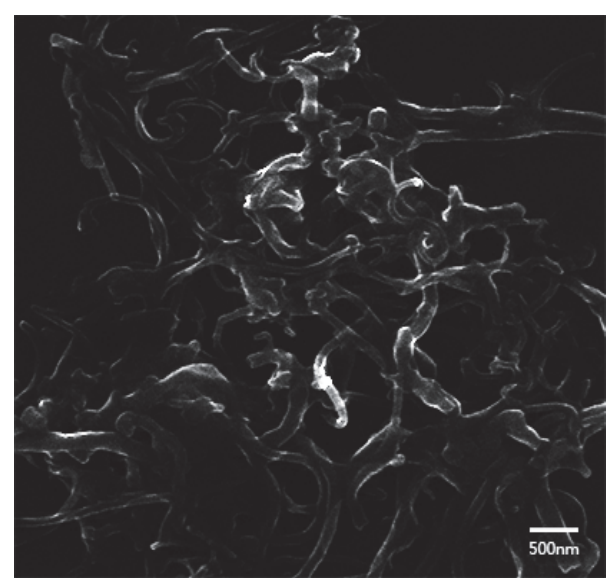

(a)

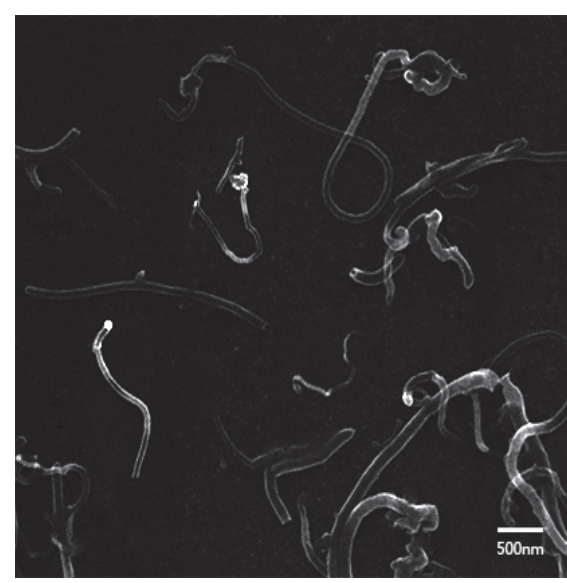

(b)

FIGURE 7: SEM images of dispersion 1-2: (a) acidity $(\mathrm{pH}=5)$; (b) alkalinity $(\mathrm{pH}=9$ ).

\section{Acknowledgments}

This work is supported by the Basic Research Project of Shanxi Province (2015021092), National Science Foundation of China (nos. 61471255, 61474079, 61501316, 51505324, and $51622507)$, and 863 project (2015AA042601).

\section{References}

[1] S. Iijima, "Helical microtubules of graphitic carbon," Nature, vol. 354, no. 6348, pp. 56-58, 1991.

[2] C.-C. Chang, I.-K. Hsu, M. Aykol, W.-H. Hung, C.-C. Chen, and S. B. Cronin, "A new lower limit for the ultimate breaking strain of carbon nanotubes," ACS Nano, vol. 4, no. 9, pp. 5095-5100, 2010.

[3] M.-F. Yu, O. Lourie, M. J. Dyer, K. Moloni, T. F. Kelly, and R. S. Ruoff, "Strength and breaking mechanism of multiwalled carbon nanotubes under tensile load," Science, vol. 287, no. 5453, pp. 637-640, 2000.

[4] S. J. Tans, A. R. M. Verschueren, and C. Dekker, "Roomtemperature transistor based on a single carbon nanotube," Nature, vol. 393, no. 6680, pp. 49-52, 1998.

[5] P.-C. Chen, S. Sukcharoenchoke, K. Ryu et al., "2,4,6Trinitrotoluene (TNT) chemical sensing based on aligned single-walled carbon nanotubes and $\mathrm{ZnO}$ nanowires," Advanced Materials, vol. 22, no. 17, pp. 1900-1904, 2010.

[6] V. Sazonova, Y. Yaish, H. Üstünel, D. Roundy, T. A. Arias, and P. L. McEuen, "A tunable carbon nanotube electromechanical oscillator," Nature, vol. 431, pp. 284-287, 2004.

[7] J. Ji, Z. Zhou, X. Yang, W. Zhang, S. Sang, and P. Li, "Onedimensional nano-interconnection formation," Small, vol. 9, no. 18, pp. 3014-3029, 2013.

[8] R. Krupke, F. Hennrich, H. B. Weber, M. M. Kappes, and H. V. Löhneysen, "Simultaneous deposition of metallic bundles of single-walled carbon nanotubes using ac-dielectrophoresis," Nano Letters, vol. 3, no. 8, pp. 1019-1023, 2003.

[9] A. Thess, R. Lee, P. Nikolaev et al., "Crystalline ropes of metallic carbon nanotubes," Science, vol. 273, no. 5274, pp. 483-487, 1996.

[10] H. M. Cheng, F. Li, G. Su et al., "Large-scale and low-cost synthesis of single-walled carbon nanotubes by the catalytic pyrolysis of hydrocarbons," Applied Physics Letters, vol. 72, no. 25, pp. 3282-3284, 1998.

[11] Z.-H. Hu, S.-M. Dong, J.-B. Hu et al., "Synthesis of carbon nanotubes on carbon fibers by modified chemical vapor deposition," New Carbon Materials, vol. 27, no. 5, pp. 352-361, 2012.

[12] P.-X. Hou, C. Liu, and H.-M. Cheng, "Purification of carbon nanotubes," Carbon, vol. 46, no. 15, pp. 2003-2025, 2008.

[13] E. Dujardin, T. W. Ebbesen, A. Krishnan, and M. M. J. Treacy, "Purification of single-shell nanotubes," Advanced Materials, vol. 10, no. 8, pp. 611-613, 1998.

[14] M. F. Islam, E. Rojas, D. M. Bergey, A. T. Johnson, and A. G. Yodh, "High weight fraction surfactant solubilization of singlewall carbon nanotubes in water," Nano Letters, vol. 3, no. 2, pp. 269-273, 2003.

[15] M. J. O’Connell, P. Boul, L. M. Ericson et al., "Reversible watersolubilization of single-walled carbon nanotubes by polymer wrapping," Chemical Physics Letters, vol. 342, no. 3-4, pp. 265271, 2001.

[16] Z. Wang, X. Chang, Z. Lu, M. Gu, Y. Zhao, and X. Gao, "A precision structural model for fullerenols," Chemical Science, vol. 5, no. 8, pp. 2940-2948, 2014.

[17] L. Y. Chiang, J. B. Bhonsle, L. Wang, S. F. Shu, T. M. Chang, and J. R. Hwu, "Efficient one-flask synthesis of water-soluble [60]fullerenols," Tetrahedron, vol. 52, no. 14, pp. 4963-4972, 1996.

[18] H. Nie, W. Guo, Y. Yuan et al., "PEGylation of double-walled carbon nanotubes for increasing their solubility in water," Nano Research, vol. 3, no. 2, pp. 103-109, 2010.

[19] W. S. Bacsa, D. Ugarte, A. Châtelain, and W. A. De Heer, "Highresolution electron microscopy and inelastic light scattering of purified multishelled carbon nanotubes," Physical Review B, vol. 50, no. 20, pp. 15473-15476, 1994.

[20] E. F. Antunes, A. O. Lobo, E. J. Corat, and V. J. Trava-Airoldi, "Influence of diameter in the Raman spectra of aligned multiwalled carbon nanotubes," Carbon, vol. 45, no. 5, pp. 913-921, 2007.

[21] Y. Kim and D. E. Luzzi, "Purification of pulsed laser synthesized single wall carbon nanotubes by magnetic filtration," Journal of Physical Chemistry B, vol. 109, no. 35, pp. 16636-16643, 2005. 
[22] S.-Y. Lee and S.-J. Park, "Hydrogen adsorption of acid-treated multi-walled carbon nanotubes at low temperature," Bulletin of the Korean Chemical Society, vol. 31, no. 6, pp. 1596-1600, 2010.

[23] J. Yu, N. Grossiord, C. E. Koning, and J. Loos, "Controlling the dispersion of multi-wall carbon nanotubes in aqueous surfactant solution," Carbon, vol. 45, no. 3, pp. 618-623, 2007.

[24] Z. Wang, Z. Lu, Y. Zhao, and X. Gao, "Oxidation-induced watersolubilization and chemical functionalization of fullerenes $\mathrm{C}_{60}$, $\mathrm{Gd} @ \mathrm{C}_{60}$ and $\mathrm{Gd} @ \mathrm{C}_{82}$ : atomistic insights into the formation mechanisms and structures of fullerenols synthesized by different methods," Nanoscale, vol. 7, no. 7, pp. 2914-2925, 2015. 

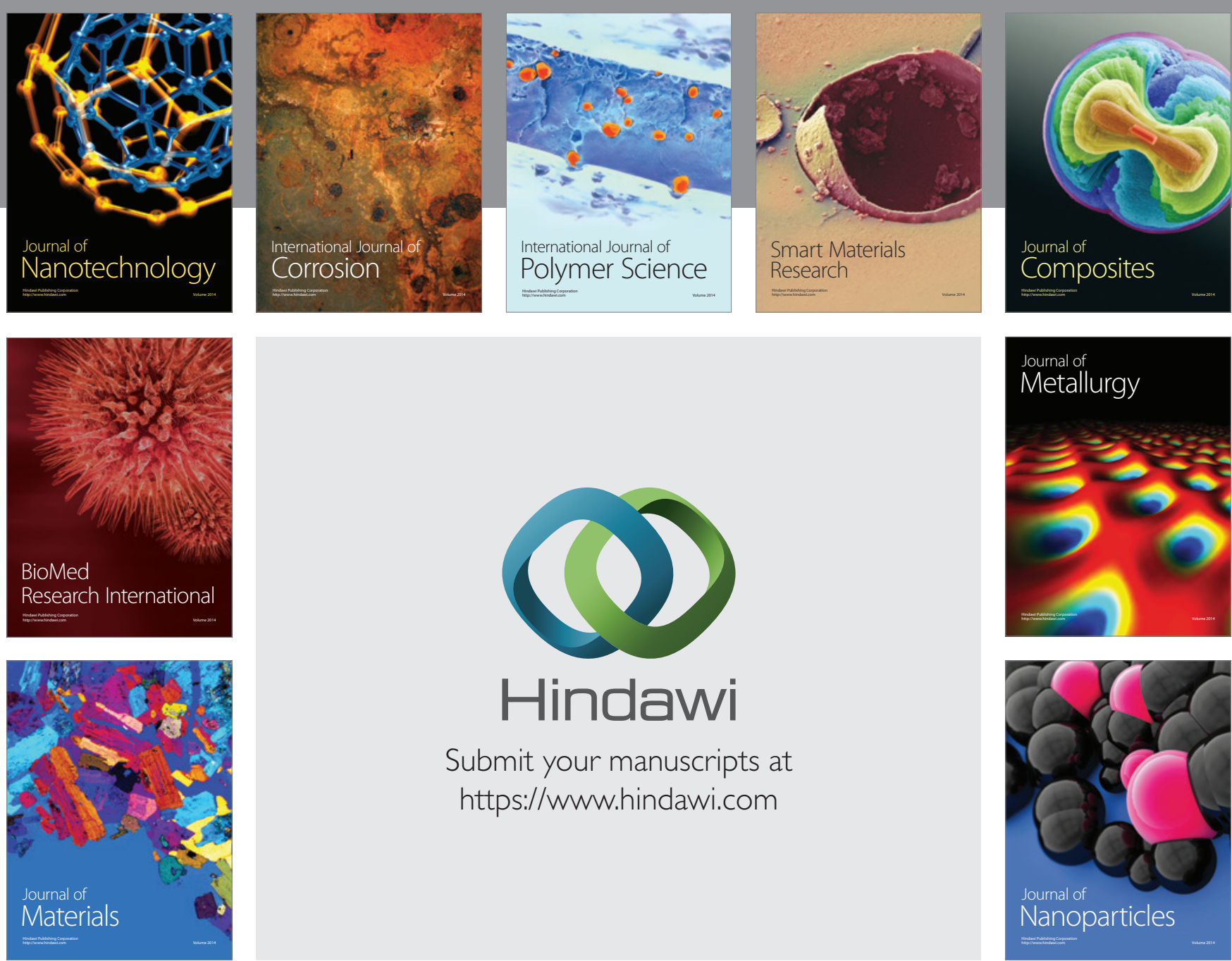

\section{Hindawi}

Submit your manuscripts at

https://www.hindawi.com

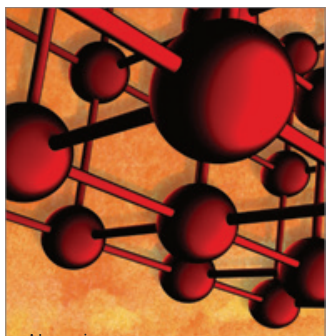

Materials Science and Engineering
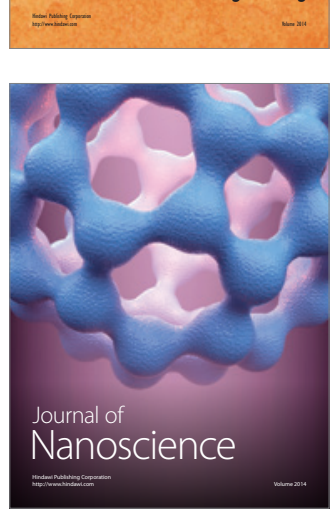
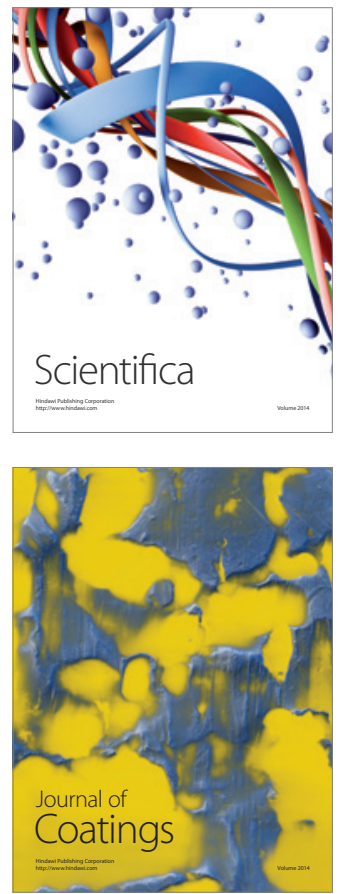
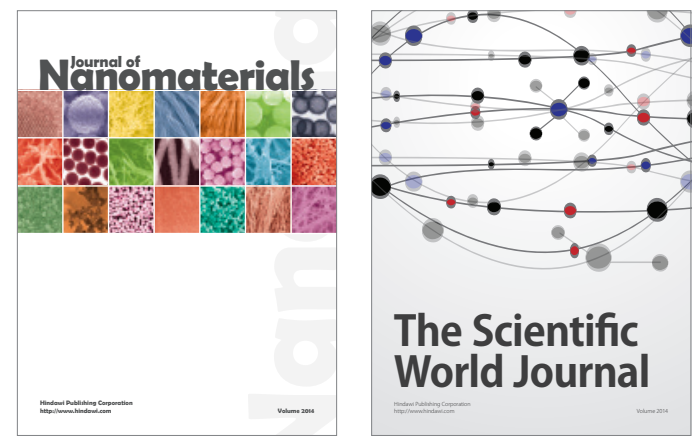

The Scientific World Journal
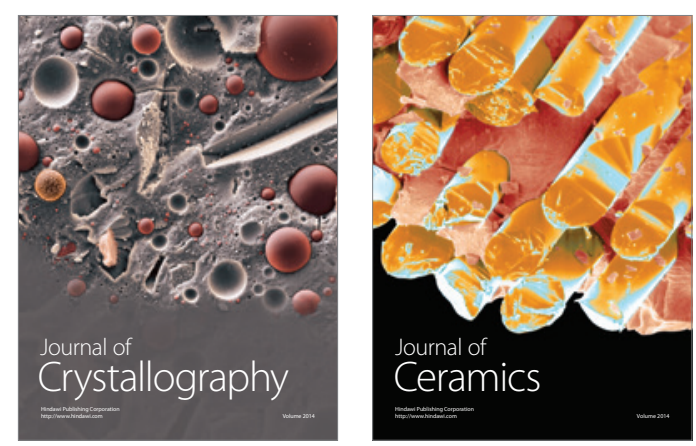
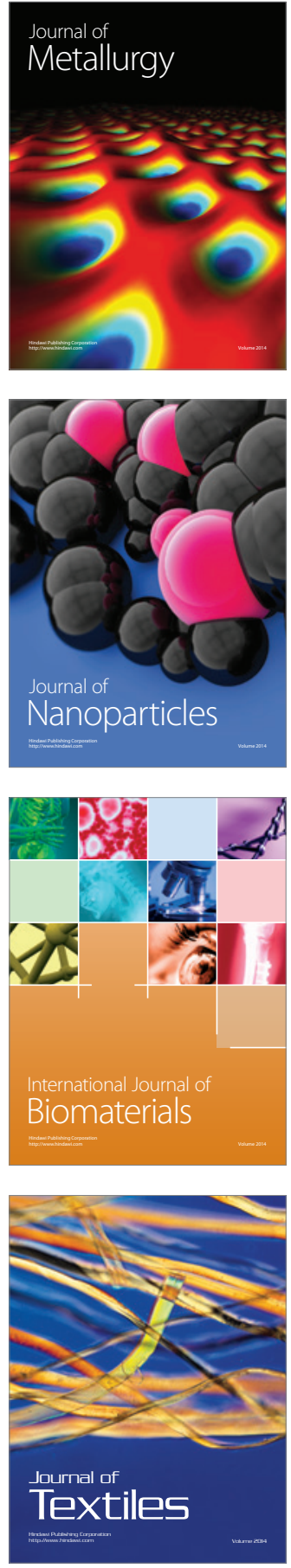